CRYSTALLOGRAPHIC COMMUNICATIONS

ISSN 2056-9890

Received 24 May 2019

Accepted 27 May 2019

Edited by J. Simpson, University of Otago, New Zealand

Keywords: crystal structure; protonated macrocycle; nitrate; hydrate; hydrogen bonds; synchrotron radiation.

CCDC reference: 1918729

Supporting information: this article has supporting information at journals.iucr.org/e

\section{Crystal structure of 3,14-diethyl-2,13-diaza-6,17- diazoniatricyclo[16.4.0.0 $\left.{ }^{7,12}\right]$ docosane dinitrate dihydrate from synchrotron $\mathrm{X}$-ray data}

\author{
Dohyun Moon, ${ }^{\text {a }}$ Sunghwan Jeon, ${ }^{\mathrm{b}}$ Keon Sang $\mathrm{Ryoo}^{\mathrm{b}}$ and Jong-Ha Choi ${ }^{\mathrm{b} *}$ \\ ${ }^{\mathbf{a} B}$ Beamline Department, Pohang Accelerator Laboratory, Pohang 37673, Republic of Korea, and ${ }^{\mathbf{b}}$ Department of \\ Chemistry, Andong National University, Andong 36729, Republic of Korea. *Correspondence e-mail: jhchoi@anu.ac.kr
}

The crystal structure of title salt, $\mathrm{C}_{22} \mathrm{H}_{46} \mathrm{~N}_{4}{ }^{2+} \cdot 2 \mathrm{NO}_{3}{ }^{-} \cdot 2 \mathrm{H}_{2} \mathrm{O}$, has been determined using synchrotron radiation at $220 \mathrm{~K}$. The structure determination reveals that protonation has occurred at diagonally opposite amine $\mathrm{N}$ atoms. The asymmetric unit contains half a centrosymmetric dication, one nitrate anion and one water molecule. The molecular dication, $\mathrm{C}_{22} \mathrm{H}_{46} \mathrm{~N}_{4}{ }^{2+}$, together with the nitrate anion and hydrate water molecule are involved in an extensive range of hydrogen bonds. The molecule is stabilized, as is the conformation of the dication, by forming intermolecular $\mathrm{N}-\mathrm{H} \cdots \mathrm{O}, \mathrm{O}-\mathrm{H} \cdots \mathrm{O}$, together with intramolecular $\mathrm{N}-\mathrm{H} \cdots \mathrm{N}$ hydrogen bonds.

\section{Chemical context}

The 3,14-diethyl-2,6,13,17-tetraazatricyclo(16.4.0.0 7,12 $)$ docosane macrocycle $\left(\mathrm{C}_{22} \mathrm{H}_{44} \mathrm{~N}_{4}, L\right)$ contains a cyclam backbone with two cyclohexane subunits. Ethyl groups are also attached to the 3 and 14 carbon atoms of the propyl chains that bridge opposite pairs of $\mathrm{N}$ atoms in the structure. The macrocyclic ligand $L$ is a strongly basic amine capable of forming the dication, $\left[\mathrm{C}_{22} \mathrm{H}_{46} \mathrm{~N}_{4}\right]^{2+}$ or the tetracation $\left[\mathrm{C}_{22} \mathrm{H}_{48} \mathrm{~N}_{4}\right]^{4+}$ in which all of the $\mathrm{N}-\mathrm{H}$ bonds are generally available for hydrogenbond formation. These di- or tetra-ammonium cations may be suitable for the removal of toxic heavy metal ions from water. The crystal structures of $[\mathrm{Cu}(L)]\left(\mathrm{ClO}_{4}\right)_{2}$ ( $\mathrm{Lim}$ et al., 2006), $[\mathrm{Cu}(L)]\left(\mathrm{NO}_{3}\right)_{2}, \quad\left[\mathrm{Cu}(L)\left(\mathrm{H}_{2} \mathrm{O}\right)_{2}\right](\mathrm{SCN})_{2}$ (Choi et al., 2012), $\left[\mathrm{Ni}(L)\left(\mathrm{NO}_{3}\right)_{2}\right]$ (Subhan \& Choi, 2014), $\left[\mathrm{Ni}(L)\left(\mathrm{N}_{3}\right)_{2}\right]$ (Lim et $a l ., 2015)$ and $\left[\mathrm{Ni}(L)(\mathrm{NCS})_{2}\right](\mathrm{Lim} \& \mathrm{Choi}, 2017)$ have been reported. In these complexes, $\mathrm{Cu}^{\mathrm{II}}$ or $\mathrm{Ni}^{\mathrm{II}}$ cations have tetragonally distorted octahedral environments with the four $\mathrm{N}$ atoms of the macrocyclic ligand in equatorial positions and the $\mathrm{O} / \mathrm{N}$ atoms of anions or water molecules in the axial positions, while $[\mathrm{Ni}(L)]\left(\mathrm{ClO}_{4}\right)_{2} \cdot 2 \mathrm{H}_{2} \mathrm{O}$ (Subhan \& Choi, 2014) has a square-planar geometry around the $\mathrm{Ni}^{\mathrm{II}}$ atom that binds to the four nitrogen atoms of the macrocyclic ligand. The macrocyclic ligands in the $\mathrm{Cu}^{\mathrm{II}}$ and $\mathrm{Ni}^{\mathrm{II}}$ complexes adopt the most stable trans-III conformation. Recently, we also reported the crystal structures of $\left[\mathrm{C}_{22} \mathrm{H}_{46} \mathrm{~N}_{4}\right]\left(\mathrm{ClO}_{4}\right)_{2}$ (Aree et al., 2018), $\left[\mathrm{C}_{22} \mathrm{H}_{46} \mathrm{~N}_{4}\right] \mathrm{Cl}_{2} \cdot 4 \mathrm{H}_{2} \mathrm{O}$ (Moon et al., 2013) and $\left(\mathrm{C}_{22} \mathrm{H}_{44} \mathrm{~N}_{4}\right)_{2} \cdot-$ $2 \mathrm{NaClO}_{4}$ (Aree et al., 2018). To further investigate the hydrogen-bonding behavior, we report here on the synthesis of a new hydrated nitrate salt, $\left[\mathrm{C}_{22} \mathrm{H}_{46} \mathrm{~N}_{4}\right]\left(\mathrm{NO}_{3}\right)_{2} \cdot 2 \mathrm{H}_{2} \mathrm{O}$, (I), and its structural characterization by synchrotron singlecrystal X-ray diffraction. 


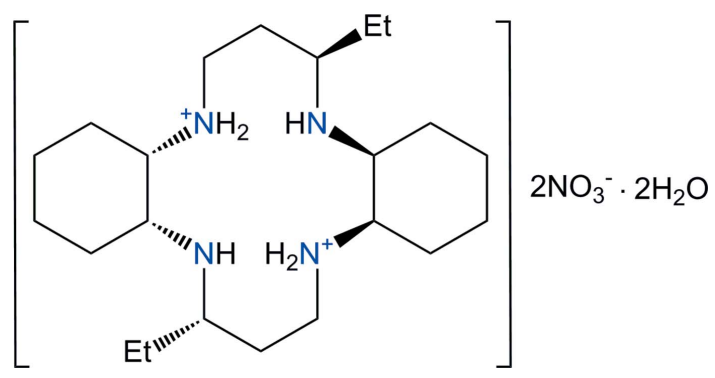

\section{Structural commentary}

The title compound has a positively charged macrocyclic dication, two nitrate anions and two solvent water molecules and was prepared during a study of the macrocyclic ligand and its silver(II) complex. An ellipsoid plot of the molecular components in compound (I) is shown in Fig. 1 along with the atom-numbering scheme. The asymmetric unit consists of one half of the macrocycle, which lies about a center of inversion, one nitrate anion and one solvent water molecule. The four $\mathrm{N}$ atoms are coplanar, and the two ethyl substituents are anti with respect to the macrocyclic plane as a result of the molecular inversion symmetry. The six-membered cyclohexane ring is in a stable chair conformation. Within the centrosymmetric diprotonated amine unit $\left[\mathrm{C}_{22} \mathrm{H}_{46} \mathrm{~N}_{4}\right]^{2+}$, the $\mathrm{C}-\mathrm{C}$ and $\mathrm{N}-\mathrm{C}$ bond lengths vary from 1.517 (2) to 1.533 (2) $\AA$ and from 1.485 (2) to 1.501 (2) $\AA$, respectively. The macrocycle is protonated at the $\mathrm{N} 2$ atom, which is similar to the situation found for $\left[\mathrm{C}_{22} \mathrm{H}_{46} \mathrm{~N}_{4}\right]\left(\mathrm{ClO}_{4}\right)_{2}$ (Aree et al., 2018), but differs from the protonation of the $\mathrm{N} 1$ atom in $\left[\mathrm{C}_{22} \mathrm{H}_{46} \mathrm{~N}_{4}\right] \mathrm{Cl}_{2} \cdot 4 \mathrm{H}_{2} \mathrm{O}$ (Moon et al., 2013). The protonation on the $\mathrm{N}$ atom might

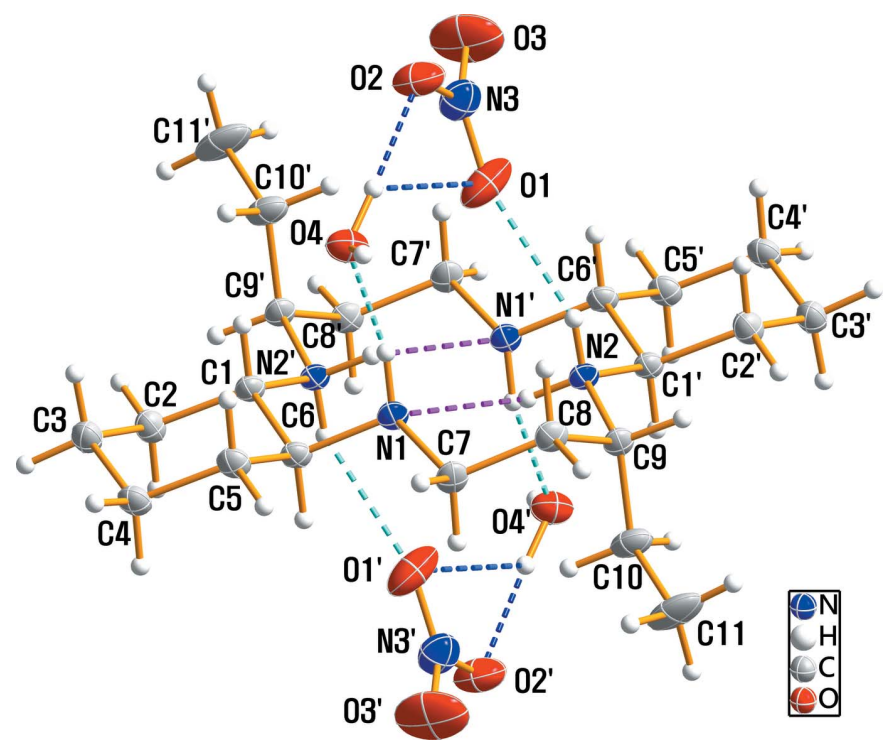

Figure 1

The molecular structure of (I), drawn with displacement ellipsoids at the $30 \%$ probability level. Primed atoms are related by the symmetry code $(1-x, 1-y, 1-z)$. Dashed lines represent $\mathrm{N}-\mathrm{H} \cdots \mathrm{O}$ (cyan), $\mathrm{N}-$ $\mathrm{H} \cdots \mathrm{N}$ (pink) and $\mathrm{O}-\mathrm{H} \cdots \mathrm{O}$ (blue) hydrogen-bonding interactions, respectively.
Table 1

Hydrogen-bond geometry $\left(\AA{ }^{\circ}\right)$.

\begin{tabular}{lllll}
\hline$D-\mathrm{H} \cdots A$ & $D-\mathrm{H}$ & $\mathrm{H} \cdots A$ & $D \cdots A$ & $D-\mathrm{H} \cdots A$ \\
\hline $\mathrm{N} 2-\mathrm{H} 2 A N \cdots \mathrm{N} 1$ & 0.90 & 2.40 & $2.9703(18)$ & 121 \\
$\mathrm{~N} 2-\mathrm{H} 2 A N \cdots \mathrm{N} 1^{\mathrm{i}}$ & 0.90 & 2.41 & $2.8141(17)$ & 107 \\
$\mathrm{~N} 1-\mathrm{H} 1 N \cdots \mathrm{O} 4$ & 0.94 & 1.84 & $2.7493(19)$ & 163 \\
$\mathrm{~N} 2-\mathrm{H} 2 A N \cdots \mathrm{N} 1$ & 0.90 & 2.40 & $2.9703(18)$ & 121 \\
$\mathrm{~N} 2-\mathrm{H} 2 B N \cdots \mathrm{O} 1$ & 0.90 & 2.27 & $3.031(2)$ & 142 \\
$\mathrm{O} 4-\mathrm{H} 1 O \cdots \mathrm{O} 1$ & $0.94(1)$ & $2.57(2)$ & $3.169(3)$ & $122(2)$ \\
$\mathrm{O} 4-\mathrm{H} 1 O \cdots \mathrm{O} 2$ & $0.94(1)$ & $1.84(1)$ & $2.768(2)$ & $174(2)$ \\
$\mathrm{O} 4-\mathrm{H} 2 O \cdots \mathrm{O} 2^{\mathrm{ii}}$ & $0.94(1)$ & $2.04(1)$ & $2.914(2)$ & $155(2)$ \\
$\mathrm{O} 4-\mathrm{H} 2 O \cdots 3^{\mathrm{ii}}$ & $0.94(1)$ & $2.31(2)$ & $3.120(4)$ & $144(2)$ \\
\hline
\end{tabular}

Symmetry codes: (i) $-x+1,-y+1,-z+1$; (ii) $x,-y+\frac{3}{2}, z-\frac{1}{2}$.

depend on the location of the acceptor atoms of the counteranion involved in hydrogen bonding. The ranges of $\mathrm{N}-\mathrm{C}-\mathrm{C}$ and $\mathrm{C}-\mathrm{N}-\mathrm{C}$ angles are $108.07(11)$ to $111.14(12)^{\circ}$ and 115.09 (11) to $115.19(10)^{\circ}$, respectively. The bond lengths and angles within the $\left[\mathrm{C}_{22} \mathrm{H}_{46} \mathrm{~N}_{4}\right]^{2+}$ dication are comparable to those found in $\left[\mathrm{C}_{22} \mathrm{H}_{46} \mathrm{~N}_{4}\right]\left(\mathrm{ClO}_{4}\right)_{2}$ (Aree et al., 2018) and $\left[\mathrm{C}_{22} \mathrm{H}_{46} \mathrm{~N}_{4}\right] \mathrm{Cl}_{2} \cdot 4 \mathrm{H}_{2} \mathrm{O}$ (Moon et al., 2013). The nitrate counteranion has a distorted trigonal-planar geometry as a result of the influence of hydrogen bonding on the $\mathrm{N}-\mathrm{O}$ bond lengths and the $\mathrm{O}-\mathrm{N}-\mathrm{O}$ angles. The $\mathrm{N}-\mathrm{O}$ bond distances range from 1.204 (3) to 1.214 (2) $\AA$ and the $\mathrm{O}-\mathrm{N}-\mathrm{O}$ angles from $117.4(2)$ to $123.1(3)^{\circ}$.

\section{Supramolecular features}

Extensive $\mathrm{N}-\mathrm{H} \cdots \mathrm{O}, \mathrm{O}-\mathrm{H} \cdots \mathrm{O}$ and $\mathrm{N}-\mathrm{H} \cdots \mathrm{N}$ hydrogenbonding interactions occur in the crystal structure (Table 1).

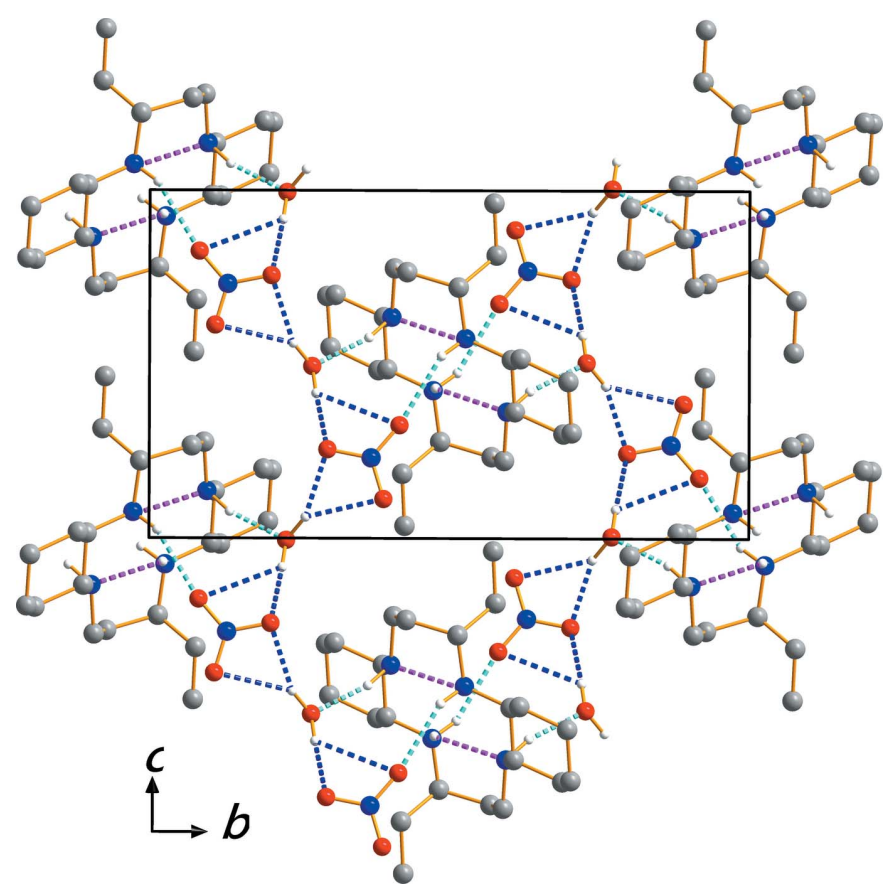

Figure 2

The crystal packing in (I), viewed perpendicular to the $b c$ plane. Dashed lines represent $\mathrm{N}-\mathrm{H} \cdots \mathrm{O}$ (cyan), $\mathrm{N}-\mathrm{H} \cdots \mathrm{N}$ (pink) and $\mathrm{O}-\mathrm{H} \cdots \mathrm{O}$ (blue) hydrogen bonding interactions, respectively. $\mathrm{H}$ atoms bound to $\mathrm{C}$ have been omitted. 
Table 2

Experimental details.

\begin{tabular}{|c|c|}
\hline \multicolumn{2}{|l|}{ Crystal data } \\
\hline Chemical formula & $\mathrm{C}_{22} \mathrm{H}_{46} \mathrm{~N}_{4}{ }^{2+} \cdot 2 \mathrm{NO}_{3}{ }^{-} \cdot 2 \mathrm{H}_{2} \mathrm{O}$ \\
\hline$M_{\mathrm{r}}$ & 526.68 \\
\hline Crystal system, space group & Monoclinic, $P 2_{1} / c$ \\
\hline Temperature $(\mathrm{K})$ & 220 \\
\hline$a, b, c(\AA)$ & $8.6420(17), 16.687$ (3), 9.7340 (19) \\
\hline$\beta\left({ }^{\circ}\right)$ & $96.46(3)$ \\
\hline$V\left(\AA^{6}\right)$ & $1394.8(5)$ \\
\hline$Z$ & 2 \\
\hline Radiation type & Synchrotron, $\lambda=0.610 \AA$ \\
\hline $\begin{array}{l}\mu\left(\mathrm{mm}^{-1}\right) \\
\text { Crystal size }(\mathrm{mm})\end{array}$ & $0.13 \times 0.09 \times 0.05$ \\
\hline \multicolumn{2}{|l|}{ Data collection } \\
\hline Diffractometer & $\begin{array}{l}\text { Rayonix MX225HS CCD area } \\
\text { detector }\end{array}$ \\
\hline Absorption correction & $\begin{array}{l}\text { Empirical (using intensity } \\
\text { measurements) (HKL3000sm } \\
\text { SCALEPACK; Otwinowski \& } \\
\text { Minor, 1997) }\end{array}$ \\
\hline$T_{\min }, T_{\max }$ & $0.919,1.000$ \\
\hline $\begin{array}{l}\text { No. of measured, independent and } \\
\text { observed }[I>2 \sigma(I)] \text { reflections }\end{array}$ & 14284, 3736, 2968 \\
\hline$R_{\mathrm{int}}$ & 0.027 \\
\hline$(\sin \theta / \lambda)_{\max }\left(\AA^{-1}\right)$ & 0.693 \\
\hline \multicolumn{2}{|l|}{ Refinement } \\
\hline$R\left[F^{2}>2 \sigma\left(F^{2}\right)\right], w R\left(F^{2}\right), S$ & $0.062,0.211,1.10$ \\
\hline No. of reflections & 3736 \\
\hline No. of parameters & 170 \\
\hline No. of restraints & 4 \\
\hline H-atom treatment & $\begin{array}{l}\mathrm{H} \text { atoms treated by a mixture of } \\
\text { independent and constrained } \\
\text { refinement }\end{array}$ \\
\hline$\Delta \rho_{\max }, \Delta \rho_{\min }\left(\mathrm{e} \AA^{-3}\right)$ & $0.73,-0.56$ \\
\hline
\end{tabular}

Computer programs: PAL BL2D-SMDC Program (Shin et al., 2016), HKL3000sm (Otwinowski \& Minor, 1997), SHELXT2018 (Sheldrick, 2015a), SHELXL2018 (Sheldrick, 2015b), DIAMOND 4 (Putz \& Brandenburg, 2014) and publCIF (Westrip, 2010).

The crystal packing viewed along the $a$ axis is shown in Fig. 2 . The $\mathrm{O}-\mathrm{H} \cdots \mathrm{O}$ hydrogen bonds link the water molecules to neighboring nitrate anions, while $\mathrm{N}-\mathrm{H} \cdots \mathrm{O}$ hydrogen bonds interconnect the $\left[\mathrm{C}_{22} \mathrm{H}_{46} \mathrm{~N}_{4}\right]^{2+}$ cations with both the nitrate anions and the water molecules. The crystal structure is stabilized by molecular hydrogen bonds involving the macrocycle $\mathrm{N}-\mathrm{H}$ groups and water $\mathrm{O}-\mathrm{H}$ groups as donors, and the $\mathrm{O}$ atoms of the water molecules and nitrate anions as acceptors, giving rise to a three-dimensional framework (Figs. 1 and 2).

\section{Database survey}

A search of the Cambridge Structural (Version 5.40, Feb 2019 with 1 update; Groom et al., 2016) gave just three hits for organic compounds containing the macrocycles $\left[\mathrm{C}_{22} \mathrm{H}_{48} \mathrm{~N}_{4}\right]^{4+}$, $\left[\mathrm{C}_{22} \mathrm{H}_{46} \mathrm{~N}_{4}\right]^{2+}$ or $\left(\mathrm{C}_{22} \mathrm{H}_{44} \mathrm{~N}_{4}\right)$. The crystal structures of $\left[\mathrm{C}_{22} \mathrm{H}_{46} \mathrm{~N}_{4}\right]\left(\mathrm{ClO}_{4}\right)_{2}$ (Aree et al., 2018), $\left[\mathrm{C}_{22} \mathrm{H}_{46} \mathrm{~N}_{4}\right] \mathrm{Cl}_{2} \cdot 4 \mathrm{H}_{2} \mathrm{O}$ (Moon et al., 2013) and $\left(\mathrm{C}_{22} \mathrm{H}_{44} \mathrm{~N}_{4}\right)_{2} \cdot 2 \mathrm{NaClO}_{4}$ (Aree et al., 2018) were reported by us previously. Until now, no crystal structures of any $\left[\mathrm{C}_{22} \mathrm{H}_{46} \mathrm{~N}_{4}\right]^{2+}$ or $\left[\mathrm{C}_{22} \mathrm{H}_{48} \mathrm{~N}_{4}\right]^{4+}$ compounds with a nitrate anion have been deposited.

\section{Synthesis and crystallization}

Commercially available trans-1,2-cyclohexanediamine, ethyl vinyl ketone and silver nitrate (Sigma-Aldrich) were used as provided. All other chemicals were reagent grade and used without further purification. As a starting material, 3,14-diethyl-2,6,13,17-tetraazatricyclo(16.4.0.0 $0^{7,12}$ )docosane, $L$ was prepared according to a published procedure (Lim et al., 2006). A solution of the macrocyclic ligand, $L(0.33 \mathrm{~g}$, $1.0 \mathrm{mmol}$ ) in methanol $10 \mathrm{~mL}$ was added dropwise to a stirred solution of $\mathrm{AgNO}_{3}(0.34 \mathrm{~g}, 2.0 \mathrm{mmol})$ in water $10 \mathrm{~mL}$. The solution turned an orange color and the metallic silver that formed was filtered off. The orange filtrate was kept in an open beaker, protected from light, at room temperature. Block-like colorless crystals of suitable for X-ray analysis were obtained unexpectedly from the solution over a period of a few weeks.

\section{Refinement}

Crystal data, data collection and structure refinement details are summarized in Table 2. All C and $\mathrm{N}$-bound $\mathrm{H}$ atoms in the complex were placed in geometrically idealized positions and constrained to ride on their parent atoms, with $\mathrm{C}-\mathrm{H}$ distances of $0.97-0.99 \AA$, and with an $\mathrm{N}-\mathrm{H}$ distance of $0.90 \AA$ with $U_{\text {iso }}(\mathrm{H})$ values of 1.2 and 1.5 times the $U_{\text {eq }}$ of the parent atoms, respectively. The $\mathrm{N}$-bound $\mathrm{H}$ atoms of the $\left[\mathrm{C}_{22} \mathrm{H}_{46} \mathrm{~N}_{4}\right]^{2+}$ cation and the $\mathrm{O}$-bound $\mathrm{H}$ atoms of the water molecules were located in a difference-Fourier map and refined isotropically, with the $\mathrm{N}-\mathrm{H}$ distance restrained using DFIX [0.9 (2) $\mathrm{A}]$ and the $\mathrm{O}-$ $\mathrm{H}$ distances and $\mathrm{H}-\mathrm{O}-\mathrm{H}$ angles restrained using DFIX and DANG constraints [0.94 (2) and $1.55(2) \AA]$, respectively.

\section{Funding information}

This work was supported by a Research Grant of Andong National University. The X-ray crystallography experiment at the PLS-II BL2D-SMC beamline was supported in part by MSICT and POSTECH.

\section{References}

Aree, T., Hong, Y. P. \& Choi, J.-H. (2018). J. Mol. Struct. 1163, 8693.

Choi, J.-H., Subhan, M. A. \& Ng, S. W. (2012). J. Coord. Chem. 65, 3481-3491.

Groom, C. R., Bruno, I. J., Lightfoot, M. P. \& Ward, S. C. (2016). Acta Cryst. B72, 171-179.

Lim, I.-T. \& Choi, K.-Y. (2017). Polyhedron, 127, 361-368.

Lim, I.-T., Kim, C.-H. \& Choi, K.-Y. (2015). Polyhedron, 100, $43-$ 48.

Lim, J. H., Kang, J. S., Kim, H. C., Koh, E. K. \& Hong, C. S. (2006). Inorg. Chem. 45, 7821-7827.

Moon, D., Subhan, M. A. \& Choi, J.-H. (2013). Acta Cryst. E69, o1620. Otwinowski, Z. \& Minor, W. (1997). Methods in Enzymology, Vol. 276, Macromolecular Crystallography, Part A, edited by C. W. Carter Jr \& R. M. Sweet, pp 307-326. New York: Academic Press.

Putz, H. \& Brandenburg, K. (2014). DIAMOND. Crystal Impact GbR, Bonn, Germany.

Sheldrick, G. M. (2015a). Acta Cryst. A71, 3-8. 
Sheldrick, G. M. (2015b). Acta Cryst. C71, 3-8.

Shin, J. W., Eom, K. \& Moon, D. (2016). J. Synchrotron Rad. 23, 369373.
Subhan, M. A. \& Choi, J.-H. (2014). Spectrochim. Acta Part A, 123, 410-415.

Westrip, S. P. (2010). J. Appl. Cryst. 43, 920-925. 


\section{supporting information}

Acta Cryst. (2019). E75, 921-924 [https://doi.org/10.1107/S2056989019007655]

Crystal structure of 3,14-diethyl-2,13-diaza-6,17-diazoniatricyclo$\left[16.4 .0 .0^{7,12}\right]$ docosane dinitrate dihydrate from synchrotron $X$-ray data

\section{Dohyun Moon, Sunghwan Jeon, Keon Sang Ryoo and Jong-Ha Choi}

Computing details

Data collection: PAL BL2D-SMDC Program (Shin et al., 2016); cell refinement: HKL3000sm (Otwinowski \& Minor, 1997); data reduction: HKL3000sm (Otwinowski \& Minor, 1997); program(s) used to solve structure: SHELXT2018 (Sheldrick, 2015a); program(s) used to refine structure: SHELXL2018 (Sheldrick, 2015b); molecular graphics:

DIAMOND 4 (Putz \& Brandenburg, 2014); software used to prepare material for publication: publCIF (Westrip, 2010).

3,14-Diethyl-2,13-diaza-6,17-diazoniatricyclo[16.4.0.0 $\left.0^{7,12}\right]$ docosane dinitrate dihydrate

\section{Crystal data}

$\mathrm{C}_{22} \mathrm{H}_{46} \mathrm{~N}_{4}{ }^{2+} \cdot 2 \mathrm{NO}_{3}{ }^{-} \cdot 2 \mathrm{H}_{2} \mathrm{O}$

$M_{r}=526.68$

Monoclinic, $P 2{ }_{1} / c$

$a=8.6420$ (17) $\AA$

$b=16.687(3) \AA$

$c=9.7340(19) \AA$

$\beta=96.46(3)^{\circ}$

$V=1394.8(5) \AA^{3}$

$Z=2$

\section{Data collection}

Rayonix MX225HS CCD area detector diffractometer

Radiation source: PLSII 2D bending magnet

$\omega$ scan

Absorption correction: empirical (using intensity measurements)

(HKL3000sm SCALEPACK; Otwinowski \& Minor, 1997)

$T_{\min }=0.919, T_{\max }=1.000$

\section{Refinement}

Refinement on $F^{2}$

Least-squares matrix: full

$R\left[F^{2}>2 \sigma\left(F^{2}\right)\right]=0.062$

$w R\left(F^{2}\right)=0.211$

$S=1.10$

3736 reflections

170 parameters

4 restraints
$F(000)=576$

$D_{\mathrm{x}}=1.254 \mathrm{Mg} \mathrm{m}^{-3}$

Synchrotron radiation, $\lambda=0.610 \AA$

Cell parameters from 46866 reflections

$\theta=0.4-33.7^{\circ}$

$\mu=0.07 \mathrm{~mm}^{-1}$

$T=220 \mathrm{~K}$

Block, colorless

$0.13 \times 0.09 \times 0.05 \mathrm{~mm}$

14284 measured reflections

3736 independent reflections

2968 reflections with $I>2 \sigma(I)$

$R_{\text {int }}=0.027$

$\theta_{\max }=25.0^{\circ}, \theta_{\min }=2.0^{\circ}$

$h=-11 \rightarrow 11$

$k=-21 \rightarrow 21$

$l=-13 \rightarrow 13$

Hydrogen site location: mixed

$\mathrm{H}$ atoms treated by a mixture of independent and constrained refinement

$w=1 /\left[\sigma^{2}\left(F_{\mathrm{o}}^{2}\right)+(0.1295 P)^{2}+0.2454 P\right]$ where $P=\left(F_{\mathrm{o}}^{2}+2 F_{\mathrm{c}}^{2}\right) / 3$

$(\Delta / \sigma)_{\max }<0.001$

$\Delta \rho_{\max }=0.73 \mathrm{e}^{-3}$

$\Delta \rho_{\min }=-0.56 \mathrm{e} \AA^{-3}$ 


\section{Special details}

Geometry. All esds (except the esd in the dihedral angle between two 1.s. planes) are estimated using the full covariance matrix. The cell esds are taken into account individually in the estimation of esds in distances, angles and torsion angles; correlations between esds in cell parameters are only used when they are defined by crystal symmetry. An approximate (isotropic) treatment of cell esds is used for estimating esds involving l.s. planes.

Fractional atomic coordinates and isotropic or equivalent isotropic displacement parameters $\left(\hat{A}^{2}\right)$

\begin{tabular}{|c|c|c|c|c|}
\hline & $x$ & $y$ & $z$ & $U_{\text {iso }} * / U_{\text {eq }}$ \\
\hline N1 & $0.51219(14)$ & $0.59626(8)$ & $0.36407(11)$ & $0.0308(3)$ \\
\hline $\mathrm{H} 1 \mathrm{~N}$ & 0.473300 & 0.634200 & 0.422800 & $0.037^{*}$ \\
\hline $\mathrm{N} 2$ & $0.28123(13)$ & $0.47313(7)$ & 0.42499 (11) & $0.0278(3)$ \\
\hline $\mathrm{H} 2 \mathrm{AN}$ & 0.385711 & 0.476834 & 0.431710 & $0.033^{*}$ \\
\hline $\mathrm{H} 2 \mathrm{BN}$ & 0.246719 & 0.513235 & 0.475356 & $0.033^{*}$ \\
\hline $\mathrm{C} 1$ & $0.76020(15)$ & $0.60393(9)$ & $0.51374(13)$ & $0.0286(3)$ \\
\hline H1 & 0.716885 & 0.647557 & 0.566781 & $0.034 *$ \\
\hline $\mathrm{C} 2$ & $0.93544(17)$ & $0.61843(10)$ & $0.51640(16)$ & $0.0365(3)$ \\
\hline $\mathrm{H} 2 \mathrm{~A}$ & 0.983763 & 0.617356 & 0.612413 & $0.044 *$ \\
\hline $\mathrm{H} 2 \mathrm{~B}$ & 0.981148 & 0.575002 & 0.466503 & $0.044^{*}$ \\
\hline $\mathrm{C} 3$ & $0.97155(18)$ & $0.69835(10)$ & $0.45121(17)$ & $0.0386(4)$ \\
\hline $\mathrm{H} 3 \mathrm{~A}$ & 0.936038 & 0.742285 & 0.506664 & $0.046^{*}$ \\
\hline H3B & 1.084368 & 0.703693 & 0.450342 & $0.046^{*}$ \\
\hline $\mathrm{C} 4$ & 0.89135 (18) & $0.70410(10)$ & $0.30389(15)$ & $0.0373(3)$ \\
\hline $\mathrm{H} 4 \mathrm{~A}$ & 0.935358 & 0.663904 & 0.245983 & $0.045^{*}$ \\
\hline H4B & 0.910323 & 0.757161 & 0.266013 & $0.045^{*}$ \\
\hline C5 & $0.71640(17)$ & $0.69051(9)$ & $0.30041(15)$ & $0.0342(3)$ \\
\hline $\mathrm{H} 5 \mathrm{~A}$ & 0.667680 & 0.692382 & 0.204553 & $0.041^{*}$ \\
\hline H5B & 0.670830 & 0.733224 & 0.352056 & $0.041^{*}$ \\
\hline C6 & $0.68403(16)$ & $0.60924(9)$ & $0.36398(13)$ & $0.0296(3)$ \\
\hline H6 & 0.726833 & 0.566354 & 0.308993 & $0.035^{*}$ \\
\hline C7 & $0.42136(18)$ & $0.59147(10)$ & $0.22379(14)$ & $0.0353(3)$ \\
\hline H7A & 0.467359 & 0.550519 & 0.168718 & $0.042 *$ \\
\hline H7B & 0.427202 & 0.643030 & 0.176434 & $0.042 *$ \\
\hline $\mathrm{C} 8$ & $0.25211(17)$ & $0.57083(9)$ & $0.23440(14)$ & $0.0337(3)$ \\
\hline H8A & 0.192762 & 0.580870 & 0.144215 & $0.040^{*}$ \\
\hline H8B & 0.212715 & 0.607652 & 0.300777 & $0.040^{*}$ \\
\hline C9 & $0.21823(17)$ & $0.48504(9)$ & $0.27811(13)$ & $0.0314(3)$ \\
\hline H9 & 0.103745 & 0.478672 & 0.271539 & $0.038^{*}$ \\
\hline $\mathrm{C} 10$ & $0.2802(3)$ & $0.42191(11)$ & $0.18588(16)$ & $0.0471(4)$ \\
\hline $\mathrm{H} 10 \mathrm{~A}$ & 0.393272 & 0.428242 & 0.189202 & $0.056^{*}$ \\
\hline H10B & 0.259978 & 0.368749 & 0.222744 & $0.056^{*}$ \\
\hline C11 & $0.2090(4)$ & $0.42595(16)$ & $0.0363(2)$ & $0.0792(8)$ \\
\hline H11A & 0.096417 & 0.427628 & 0.032597 & $0.119^{*}$ \\
\hline H11B & 0.239639 & 0.378996 & -0.012820 & $0.119^{*}$ \\
\hline $\mathrm{H} 11 \mathrm{C}$ & 0.245453 & 0.473817 & -0.006600 & $0.119 *$ \\
\hline N3 & $0.2912(2)$ & $0.63006(12)$ & 0.76865 (18) & $0.0581(5)$ \\
\hline $\mathrm{O} 1$ & $0.3086(2)$ & $0.58294(10)$ & $0.6746(2)$ & $0.0812(6)$ \\
\hline $\mathrm{O} 2$ & $0.2872(2)$ & $0.70307(10)$ & $0.74420(17)$ & $0.0696(5)$ \\
\hline
\end{tabular}




$\begin{array}{lllll}\mathrm{O} 3 & 0.2875(5) & 0.6094(2) & 0.8868(3) & 0.1636(16) \\ \mathrm{O} 4 & 0.42576(16) & 0.72874(9) & 0.50503(14) & 0.0501(3) \\ \mathrm{H} 1 \mathrm{O} & 0.373(3) & 0.7221(16) & 0.5829(17) & 0.075^{*} \\ \mathrm{H} 2 \mathrm{O} & 0.366(3) & 0.7605(14) & 0.439(2) & 0.075^{*}\end{array}$

Atomic displacement parameters $\left(\AA^{2}\right)$

\begin{tabular}{lllllll}
\hline & $U^{11}$ & $U^{22}$ & $U^{33}$ & $U^{12}$ & $U^{13}$ & $U^{23}$ \\
\hline $\mathrm{N} 1$ & $0.0327(6)$ & $0.0418(7)$ & $0.0171(5)$ & $-0.0017(5)$ & $-0.0003(4)$ & $0.0035(4)$ \\
$\mathrm{N} 2$ & $0.0303(6)$ & $0.0363(6)$ & $0.0163(5)$ & $-0.0001(4)$ & $-0.0003(4)$ & $0.0006(4)$ \\
$\mathrm{C} 1$ & $0.0300(6)$ & $0.0370(7)$ & $0.0185(6)$ & $-0.0006(5)$ & $0.0012(5)$ & $0.0007(5)$ \\
$\mathrm{C} 2$ & $0.0303(7)$ & $0.0486(9)$ & $0.0302(7)$ & $-0.0013(6)$ & $0.0012(5)$ & $0.0050(6)$ \\
$\mathrm{C} 3$ & $0.0361(7)$ & $0.0496(9)$ & $0.0305(7)$ & $-0.0078(6)$ & $0.0055(6)$ & $0.0016(6)$ \\
$\mathrm{C} 4$ & $0.0380(7)$ & $0.0482(9)$ & $0.0269(7)$ & $-0.0029(6)$ & $0.0090(6)$ & $0.0038(6)$ \\
$\mathrm{C} 5$ & $0.0368(7)$ & $0.0423(8)$ & $0.0240(6)$ & $-0.0006(5)$ & $0.0048(5)$ & $0.0065(5)$ \\
$\mathrm{C} 6$ & $0.0317(6)$ & $0.0389(7)$ & $0.0180(6)$ & $0.0007(5)$ & $0.0024(5)$ & $0.0015(5)$ \\
C7 & $0.0393(8)$ & $0.0476(8)$ & $0.0176(6)$ & $-0.0062(6)$ & $-0.0027(5)$ & $0.0057(5)$ \\
C8 & $0.0344(7)$ & $0.0437(8)$ & $0.0213(6)$ & $0.0024(5)$ & $-0.0040(5)$ & $0.0046(5)$ \\
C9 & $0.0329(7)$ & $0.0430(8)$ & $0.0172(6)$ & $-0.0033(5)$ & $-0.0013(5)$ & $0.0010(5)$ \\
C10 & $0.0721(12)$ & $0.0461(10)$ & $0.0240(7)$ & $-0.0041(8)$ & $0.0097(7)$ & $-0.0044(6)$ \\
C11 & $0.141(3)$ & $0.0743(15)$ & $0.0214(8)$ & $-0.0135(15)$ & $0.0046(11)$ & $-0.0093(9)$ \\
N3 & $0.0674(11)$ & $0.0633(11)$ & $0.0418(9)$ & $-0.0045(8)$ & $-0.0025(8)$ & $0.0016(7)$ \\
O1 & $0.1056(14)$ & $0.0553(10)$ & $0.0739(12)$ & $0.0074(8)$ & $-0.0284(10)$ & $-0.0228(8)$ \\
O2 & $0.1060(14)$ & $0.0545(9)$ & $0.0509(9)$ & $0.0048(8)$ & $0.0204(9)$ & $-0.0098(6)$ \\
O3 & $0.285(5)$ & $0.140(3)$ & $0.0760(18)$ & $0.010(3)$ & $0.063(2)$ & $0.0501(17)$ \\
O4 & $0.0555(8)$ & $0.0574(8)$ & $0.0384(7)$ & $0.0082(6)$ & $0.0088(6)$ & $-0.0009(5)$ \\
& & & & & &
\end{tabular}

Geometric parameters $\left(A,{ }^{\circ}\right)$

\begin{tabular}{llll}
\hline $\mathrm{N} 1-\mathrm{C} 7$ & $1.4987(17)$ & $\mathrm{C} 5-\mathrm{H} 5 \mathrm{~B}$ & 0.9800 \\
$\mathrm{~N} 1-\mathrm{C} 6$ & $1.5009(18)$ & $\mathrm{C} 6-\mathrm{H} 6$ & 0.9900 \\
$\mathrm{~N} 1-\mathrm{H} 1 \mathrm{~N}$ & 0.94 & $\mathrm{C} 7-\mathrm{C} 8$ & $1.517(2)$ \\
$\mathrm{N} 2-\mathrm{C} 1^{\mathrm{i}}$ & $1.4784(18)$ & $\mathrm{C} 7-\mathrm{H} 7 \mathrm{~A}$ & 0.9800 \\
$\mathrm{~N} 2-\mathrm{C} 9$ & $1.4850(16)$ & $\mathrm{C} 7-\mathrm{H} 7 \mathrm{~B}$ & 0.9800 \\
$\mathrm{~N} 2-\mathrm{H} 2 \mathrm{AN}$ & 0.9000 & $\mathrm{C} 8-\mathrm{C} 9$ & $1.531(2)$ \\
$\mathrm{N} 2-\mathrm{H} 2 \mathrm{BN}$ & 0.9000 & $\mathrm{C} 8-\mathrm{H} 8 \mathrm{~A}$ & 0.9800 \\
$\mathrm{C} 1-\mathrm{C} 2$ & $1.5309(19)$ & $\mathrm{C} 8-\mathrm{H} 8 \mathrm{~B}$ & 0.9800 \\
$\mathrm{C} 1-\mathrm{C} 6$ & $1.5330(18)$ & $\mathrm{C} 9-\mathrm{C} 10$ & $1.520(2)$ \\
$\mathrm{C} 1-\mathrm{H} 1$ & 0.9900 & $\mathrm{C} 9-\mathrm{H} 9$ & 0.9900 \\
$\mathrm{C} 2-\mathrm{C} 3$ & $1.524(2)$ & $\mathrm{C} 10-\mathrm{C} 11$ & $1.517(3)$ \\
$\mathrm{C} 2-\mathrm{H} 2 \mathrm{~A}$ & 0.9800 & $\mathrm{C} 10-\mathrm{H} 10 \mathrm{~A}$ & 0.9800 \\
$\mathrm{C} 2-\mathrm{H} 2 \mathrm{~B}$ & 0.9800 & $\mathrm{C} 10-\mathrm{H} 10 \mathrm{~B}$ & 0.9800 \\
$\mathrm{C} 3-\mathrm{C} 4$ & $1.524(2)$ & $\mathrm{C} 11-\mathrm{H} 11 \mathrm{~A}$ & 0.9700 \\
$\mathrm{C} 3-\mathrm{H} 3 \mathrm{~A}$ & 0.9800 & $\mathrm{C} 11-\mathrm{H} 11 \mathrm{~B}$ & 0.9700 \\
$\mathrm{C} 3-\mathrm{H} 3 \mathrm{~B}$ & 0.9800 & $\mathrm{C} 11-\mathrm{H} 11 \mathrm{C}$ & 0.9700 \\
$\mathrm{C} 4-\mathrm{C} 5$ & $1.525(2)$ & $\mathrm{N} 3-\mathrm{O} 3$ & $1.204(3)$ \\
$\mathrm{C} 4-\mathrm{H} 4 \mathrm{~A}$ & 0.9800 & $\mathrm{~N} 3-\mathrm{O} 1$ & $1.229(3)$ \\
$\mathrm{C} 4-\mathrm{H} 4 \mathrm{~B}$ & 0.9800 & $\mathrm{~N} 3-\mathrm{O} 2$ & $1.241(2)$
\end{tabular}




\begin{tabular}{|c|c|c|c|}
\hline $\mathrm{C} 5-\mathrm{C} 6$ & $1.529(2)$ & $\mathrm{O} 4-\mathrm{H} 1 \mathrm{O}$ & $0.937(10)$ \\
\hline $\mathrm{C} 5-\mathrm{H} 5 \mathrm{~A}$ & 0.9800 & $\mathrm{O} 4-\mathrm{H} 2 \mathrm{O}$ & $0.941(9)$ \\
\hline $\mathrm{C} 7-\mathrm{N} 1-\mathrm{C} 6$ & $115.09(11)$ & $\mathrm{N} 1-\mathrm{C} 6-\mathrm{C} 1$ & $108.07(11)$ \\
\hline $\mathrm{C} 7-\mathrm{N} 1-\mathrm{H} 1 \mathrm{~N}$ & 114 & $\mathrm{C} 5-\mathrm{C} 6-\mathrm{C} 1$ & $110.95(12)$ \\
\hline $\mathrm{C} 6-\mathrm{N} 1-\mathrm{H} 1 \mathrm{~N}$ & 109 & $\mathrm{~N} 1-\mathrm{C} 6-\mathrm{H} 6$ & 109.0 \\
\hline $\mathrm{C} 1-\mathrm{i} 2-\mathrm{C} 9$ & $115.19(10)$ & $\mathrm{C} 5-\mathrm{C} 6-\mathrm{H} 6$ & 109.0 \\
\hline $\mathrm{C} 1{ }^{\mathrm{i}}-\mathrm{N} 2-\mathrm{H} 2 \mathrm{AN}$ & 108.5 & $\mathrm{C} 1-\mathrm{C} 6-\mathrm{H} 6$ & 109.0 \\
\hline $\mathrm{C} 9-\mathrm{N} 2-\mathrm{H} 2 \mathrm{AN}$ & 108.5 & $\mathrm{~N} 1-\mathrm{C} 7-\mathrm{C} 8$ & $111.11(11)$ \\
\hline $\mathrm{C} 11^{\mathrm{i}}-\mathrm{N} 2-\mathrm{H} 2 \mathrm{BN}$ & 108.5 & $\mathrm{~N} 1-\mathrm{C} 7-\mathrm{H} 7 \mathrm{~A}$ & 109.4 \\
\hline $\mathrm{C} 9-\mathrm{N} 2-\mathrm{H} 2 \mathrm{BN}$ & 108.5 & $\mathrm{C} 8-\mathrm{C} 7-\mathrm{H} 7 \mathrm{~A}$ & 109.4 \\
\hline $\mathrm{H} 2 \mathrm{AN}-\mathrm{N} 2-\mathrm{H} 2 \mathrm{BN}$ & 107.5 & $\mathrm{~N} 1-\mathrm{C} 7-\mathrm{H} 7 \mathrm{~B}$ & 109.4 \\
\hline $\mathrm{N} 22^{\mathrm{i}}-\mathrm{C} 1-\mathrm{C} 2$ & $114.50(12)$ & $\mathrm{C} 8-\mathrm{C} 7-\mathrm{H} 7 \mathrm{~B}$ & 109.4 \\
\hline $\mathrm{N} 2 \mathrm{i}-\mathrm{C} 1-\mathrm{C} 6$ & $109.65(11)$ & $\mathrm{H} 7 \mathrm{~A}-\mathrm{C} 7-\mathrm{H} 7 \mathrm{~B}$ & 108.0 \\
\hline $\mathrm{C} 2-\mathrm{C} 1-\mathrm{C} 6$ & $108.97(11)$ & $\mathrm{C} 7-\mathrm{C} 8-\mathrm{C} 9$ & $116.55(12)$ \\
\hline $\mathrm{N} 2 \mathrm{i}-\mathrm{C} 1-\mathrm{H} 1$ & 107.8 & $\mathrm{C} 7-\mathrm{C} 8-\mathrm{H} 8 \mathrm{~A}$ & 108.2 \\
\hline $\mathrm{C} 2-\mathrm{C} 1-\mathrm{H} 1$ & 107.8 & $\mathrm{C} 9-\mathrm{C} 8-\mathrm{H} 8 \mathrm{~A}$ & 108.2 \\
\hline $\mathrm{C} 6-\mathrm{C} 1-\mathrm{H} 1$ & 107.8 & $\mathrm{C} 7-\mathrm{C} 8-\mathrm{H} 8 \mathrm{~B}$ & 108.2 \\
\hline $\mathrm{C} 3-\mathrm{C} 2-\mathrm{C} 1$ & $112.32(12)$ & $\mathrm{C} 9-\mathrm{C} 8-\mathrm{H} 8 \mathrm{~B}$ & 108.2 \\
\hline $\mathrm{C} 3-\mathrm{C} 2-\mathrm{H} 2 \mathrm{~A}$ & 109.1 & $\mathrm{H} 8 \mathrm{~A}-\mathrm{C} 8-\mathrm{H} 8 \mathrm{~B}$ & 107.3 \\
\hline $\mathrm{C} 1-\mathrm{C} 2-\mathrm{H} 2 \mathrm{~A}$ & 109.1 & $\mathrm{~N} 2-\mathrm{C} 9-\mathrm{C} 10$ & $111.14(12)$ \\
\hline $\mathrm{C} 3-\mathrm{C} 2-\mathrm{H} 2 \mathrm{~B}$ & 109.1 & $\mathrm{~N} 2-\mathrm{C} 9-\mathrm{C} 8$ & $109.38(11)$ \\
\hline $\mathrm{C} 1-\mathrm{C} 2-\mathrm{H} 2 \mathrm{~B}$ & 109.1 & $\mathrm{C} 10-\mathrm{C} 9-\mathrm{C} 8$ & $113.13(13)$ \\
\hline $\mathrm{H} 2 \mathrm{~A}-\mathrm{C} 2-\mathrm{H} 2 \mathrm{~B}$ & 107.9 & $\mathrm{~N} 2-\mathrm{C} 9-\mathrm{H} 9$ & 107.7 \\
\hline $\mathrm{C} 4-\mathrm{C} 3-\mathrm{C} 2$ & $110.74(13)$ & $\mathrm{C} 10-\mathrm{C} 9-\mathrm{H} 9$ & 107.7 \\
\hline $\mathrm{C} 4-\mathrm{C} 3-\mathrm{H} 3 \mathrm{~A}$ & 109.5 & $\mathrm{C} 8-\mathrm{C} 9-\mathrm{H} 9$ & 107.7 \\
\hline $\mathrm{C} 2-\mathrm{C} 3-\mathrm{H} 3 \mathrm{~A}$ & 109.5 & $\mathrm{C} 11-\mathrm{C} 10-\mathrm{C} 9$ & $113.86(17)$ \\
\hline $\mathrm{C} 4-\mathrm{C} 3-\mathrm{H} 3 \mathrm{~B}$ & 109.5 & $\mathrm{C} 11-\mathrm{C} 10-\mathrm{H} 10 \mathrm{~A}$ & 108.8 \\
\hline $\mathrm{C} 2-\mathrm{C} 3-\mathrm{H} 3 \mathrm{~B}$ & 109.5 & $\mathrm{C} 9-\mathrm{C} 10-\mathrm{H} 10 \mathrm{~A}$ & 108.8 \\
\hline $\mathrm{H} 3 \mathrm{~A}-\mathrm{C} 3-\mathrm{H} 3 \mathrm{~B}$ & 108.1 & $\mathrm{C} 11-\mathrm{C} 10-\mathrm{H} 10 \mathrm{~B}$ & 108.8 \\
\hline $\mathrm{C} 3-\mathrm{C} 4-\mathrm{C} 5$ & $110.87(12)$ & $\mathrm{C} 9-\mathrm{C} 10-\mathrm{H} 10 \mathrm{~B}$ & 108.8 \\
\hline $\mathrm{C} 3-\mathrm{C} 4-\mathrm{H} 4 \mathrm{~A}$ & 109.5 & $\mathrm{H} 10 \mathrm{~A}-\mathrm{C} 10-\mathrm{H} 10 \mathrm{~B}$ & 107.7 \\
\hline $\mathrm{C} 5-\mathrm{C} 4-\mathrm{H} 4 \mathrm{~A}$ & 109.5 & $\mathrm{C} 10-\mathrm{C} 11-\mathrm{H} 11 \mathrm{~A}$ & 109.5 \\
\hline $\mathrm{C} 3-\mathrm{C} 4-\mathrm{H} 4 \mathrm{~B}$ & 109.5 & $\mathrm{C} 10-\mathrm{C} 11-\mathrm{H} 11 \mathrm{~B}$ & 109.5 \\
\hline $\mathrm{C} 5-\mathrm{C} 4-\mathrm{H} 4 \mathrm{~B}$ & 109.5 & $\mathrm{H} 11 \mathrm{~A}-\mathrm{C} 11-\mathrm{H} 11 \mathrm{~B}$ & 109.5 \\
\hline $\mathrm{H} 4 \mathrm{~A}-\mathrm{C} 4-\mathrm{H} 4 \mathrm{~B}$ & 108.1 & $\mathrm{C} 10-\mathrm{C} 11-\mathrm{H} 11 \mathrm{C}$ & 109.5 \\
\hline $\mathrm{C} 4-\mathrm{C} 5-\mathrm{C} 6$ & $110.41(12)$ & $\mathrm{H} 11 \mathrm{~A}-\mathrm{C} 11-\mathrm{H} 11 \mathrm{C}$ & 109.5 \\
\hline $\mathrm{C} 4-\mathrm{C} 5-\mathrm{H} 5 \mathrm{~A}$ & 109.6 & $\mathrm{H} 11 \mathrm{~B}-\mathrm{C} 11-\mathrm{H} 11 \mathrm{C}$ & 109.5 \\
\hline $\mathrm{C} 6-\mathrm{C} 5-\mathrm{H} 5 \mathrm{~A}$ & 109.6 & $\mathrm{O} 3-\mathrm{N} 3-\mathrm{O} 1$ & $123.1(3)$ \\
\hline $\mathrm{C} 4-\mathrm{C} 5-\mathrm{H} 5 \mathrm{~B}$ & 109.6 & $\mathrm{O} 3-\mathrm{N} 3-\mathrm{O} 2$ & $117.4(2)$ \\
\hline $\mathrm{C} 6-\mathrm{C} 5-\mathrm{H} 5 \mathrm{~B}$ & 109.6 & $\mathrm{O} 1-\mathrm{N} 3-\mathrm{O} 2$ & $119.23(19)$ \\
\hline $\mathrm{H} 5 \mathrm{~A}-\mathrm{C} 5-\mathrm{H} 5 \mathrm{~B}$ & 108.1 & $\mathrm{H} 1 \mathrm{O}-\mathrm{O} 4-\mathrm{H} 2 \mathrm{O}$ & $109.8(18)$ \\
\hline $\mathrm{N} 1-\mathrm{C} 6-\mathrm{C} 5$ & $110.75(11)$ & & \\
\hline $\mathrm{N} 2 \mathrm{i}-\mathrm{C} 1-\mathrm{C} 2-\mathrm{C} 3$ & $179.62(12)$ & $\mathrm{N} 2{ }^{\mathrm{i}}-\mathrm{C} 1-\mathrm{C} 6-\mathrm{C} 5$ & $176.50(11)$ \\
\hline $\mathrm{C} 6-\mathrm{C} 1-\mathrm{C} 2-\mathrm{C} 3$ & $56.43(16)$ & $\mathrm{C} 2-\mathrm{C} 1-\mathrm{C} 6-\mathrm{C} 5$ & $-57.46(15)$ \\
\hline $\mathrm{C} 1-\mathrm{C} 2-\mathrm{C} 3-\mathrm{C} 4$ & $-55.92(17)$ & $\mathrm{C} 6-\mathrm{N} 1-\mathrm{C} 7-\mathrm{C} 8$ & $174.85(12)$ \\
\hline $\mathrm{C} 2-\mathrm{C} 3-\mathrm{C} 4-\mathrm{C} 5$ & $55.43(18)$ & $\mathrm{N} 1-\mathrm{C} 7-\mathrm{C} 8-\mathrm{C} 9$ & $-71.31(16)$ \\
\hline
\end{tabular}




$\begin{array}{llll}\mathrm{C} 3-\mathrm{C} 4-\mathrm{C} 5-\mathrm{C} 6 & -57.05(17) & \mathrm{C} 1-\mathrm{N} 2-\mathrm{C} 9-\mathrm{C} 10 & -61.66(16) \\ \mathrm{C} 7-\mathrm{N} 1-\mathrm{C} 6-\mathrm{C} 5 & 64.03(15) & \mathrm{C} 1-\mathrm{N} 2-\mathrm{C} 9-\mathrm{C} 8 & 172.72(11) \\ \mathrm{C} 7-\mathrm{N} 1-\mathrm{C} 6-\mathrm{C} 1 & -174.25(12) & \mathrm{C} 7-\mathrm{C} 8-\mathrm{C} 9-\mathrm{N} 2 & 68.87(16) \\ \mathrm{C} 4-\mathrm{C} 5-\mathrm{C} 6-\mathrm{N} 1 & 178.64(11) & \mathrm{C} 7-\mathrm{C} 8-\mathrm{C} 9-\mathrm{C} 10 & -55.60(17) \\ \mathrm{C} 4-\mathrm{C} 5-\mathrm{C} 6-\mathrm{C} 1 & 58.63(16) & \mathrm{N} 2-\mathrm{C} 9-\mathrm{C} 10-\mathrm{C} 11 & 174.71(16) \\ \mathrm{N} 2-\mathrm{C} 1-\mathrm{C} 6-\mathrm{N} 1 & 54.91(14) & \mathrm{C} 8-\mathrm{C} 9-\mathrm{C} 10-\mathrm{C} 11 & -61.8(2) \\ \mathrm{C} 2-\mathrm{C} 1-\mathrm{C} 6-\mathrm{N} 1 & -179.05(12) & & \end{array}$

Symmetry code: (i) $-x+1,-y+1,-z+1$.

Hydrogen-bond geometry $\left(A,{ }^{\circ}\right)$

\begin{tabular}{lllll}
\hline$D-\mathrm{H} \cdots A$ & $D-\mathrm{H}$ & $\mathrm{H} \cdots A$ & $D \cdots A$ & $D-\mathrm{H} \cdots A$ \\
\hline $\mathrm{N} 2-\mathrm{H} 2 A N \cdots \mathrm{N} 1$ & 0.90 & 2.40 & $2.9703(18)$ & 121 \\
$\mathrm{~N} 2-\mathrm{H} 2 A N \cdots \mathrm{N} 1^{\mathrm{i}}$ & 0.90 & 2.41 & $2.8141(17)$ & 107 \\
$\mathrm{~N} 1-\mathrm{H} 1 N \cdots \mathrm{O} 4$ & 0.94 & 1.84 & $2.7493(19)$ & 163 \\
$\mathrm{~N} 2-\mathrm{H} 2 A N \cdots \mathrm{N} 1$ & 0.90 & 2.40 & $2.9703(18)$ & 121 \\
$\mathrm{~N} 2-\mathrm{H} 2 B N \cdots \mathrm{O} 1$ & 0.90 & 2.27 & $3.031(2)$ & 142 \\
$\mathrm{O} 4-\mathrm{H} 1 O \cdots \mathrm{O} 1$ & $0.94(1)$ & $2.57(2)$ & $3.169(3)$ & $122(2)$ \\
$\mathrm{O} 4-\mathrm{H} 1 O \cdots \mathrm{O} 2$ & $0.94(1)$ & $1.84(1)$ & $2.768(2)$ & $174(2)$ \\
$\mathrm{O} 4-\mathrm{H} 2 O \cdots \mathrm{O} 2^{\mathrm{ii}}$ & $0.94(1)$ & $2.04(1)$ & $2.914(2)$ & $155(2)$ \\
$\mathrm{O} 4-\mathrm{H} 2 O \cdots \mathrm{O} 3^{3 i}$ & $0.94(1)$ & $2.31(2)$ & $3.120(4)$ & $144(2)$
\end{tabular}

Symmetry codes: (i) $-x+1,-y+1,-z+1$; (ii) $x,-y+3 / 2, z-1 / 2$. 